\title{
A Qualitative Study of the Effects of Hearing Loss and Hearing Aid Use on Music Perception in Performing Musicians
}

DOI: $10.3766 /$ jaaa.17019

\author{
Jonathan M. Vaisberg*† \\ Ashley T. Martindale*† \\ Paula Folkeard* \\ Cathy Benedict
}

\begin{abstract}
Background: Hearing aids (HAs) are important for the rehabilitation of individuals with hearing loss. Although the rehabilitation of speech communication is well understood, less attention has been devoted to understanding hearing-impaired instrumentalists' needs to actively participate in music. Despite efforts to adjust HA settings for music acoustics, there lacks an understanding of instrumentalists' needs and if those HA adjustments satisfy their needs.
\end{abstract}

Purpose: The purpose of the current study was to explore the challenges that adult HA-wearing instrumentalists face, which prevent them from listening, responding to, and performing music.

Research Design: A qualitative methodology was employed with the use of semistructured interviews conducted with adult amateur instrumentalists.

Study Sample: Twelve HA users who were amateur ensemble instrumentalists (playing instruments from the percussion, wind, reed, brass, and string families) and between the ages of 55 and 83 years (seven men and five women) provided data for analysis in this study. Amateur in this context was defined as one who engaged mindfully in pursuit of an activity.

Data Collection and Analysis: Semistructured interviews were conducted using an open-ended interview guide. Interviews were recorded and transcribed verbatim. Transcripts were analyzed using conventional qualitative content analysis.

Results: Three categories emerged from the data: (1) participatory needs, (2) effects of HA use, and (3) effects of hearing loss. Participants primarily used HAs to hear the conductor's instructions to meaningfully participate in music rehearsals. Effects of HA use fell within two subcategories: HA music sound quality and use of an HA music program. The effects of hearing loss fell within three subcategories: inability to identify missing information, affected music components, and nonauditory music perception strategies.

Conclusions: Not surprisingly, hearing-impaired instrumentalists face challenges participating in their music activities. However, although participants articulated ways in which HAs and hearing loss affect music perception, which in turn revealed perspectives toward listening using the auditory system and other sensory systems, the primary motivation for their HA use was the need to hear the conductor's directions. These findings suggest that providing hearing-impaired instrumentalists access to musical experience via participation should be prioritized above restoring the perception of musical descriptors. Future research is needed with instrumentalists who no longer listen to or perform music because of

*Faculty of Health Sciences, National Centre for Audiology, Western University, London, ON, Canada; †Graduate Program in Health \& Rehabilitation Sciences (Hearing Science), Faculty of Health Sciences, Western University, London, ON, Canada; $\ddagger$ Don Wright Faculty of Music, Western University, London, ON, Canada

Corresponding author: Jonathan M. Vaisberg, National Centre for Audiology, Western University, London, ON, Canada N6G 1H1; Email: jvaisber@uwo.ca

The authors appreciate support from a pilot study grant from the Music Learning Across the Lifespan initiative, made possible by Research Western and the Don Wright Faculty of Music, Western University. The authors also acknowledge support for project implementation and testing of participants from the Translational Research Unit at Western University's National Centre for Audiology. 
hearing loss, so that the relationship between musical auditory deficiencies and participation can be better explored.

Key Words: hearing aids, hearing loss, instrumentalists, music perception, musicians, patient perspective, qualitative

Abbreviations: ANR = adaptive noise reduction; $\mathrm{HA}=$ hearing aid; $\mathrm{HL}=$ hearing level; PTA3 3-frequency pure-tone average; SD = standard deviation; VolP = voice-over-internet-protocol; WDRC = wide dynamic range compression

\section{INTRODUCTION}

$\mathrm{T}$ he use of hearing aids (HAs) has always been associated with improved health-related quality of life (Chisolm et al, 2007; Contrera et al, 2016), including, but not limited to, "improvements in the social, emotional, psychological, and physical well-being of people" (Said, 2017). Although the audiology community effectively understands and addresses listeners' needs for speech communication, less attention has been devoted to understanding hearing-impaired instrumentalists' needs associated with listening, responding to, and performing music. Even with a recent surge in studies investigating how hearing aid signal processing affects hearing music, there still is lack of understanding of hearing-impaired instrumentalists' needs to meaningfully listen, respond to, and perform music while wearing HAs. The central focus of this study, therefore, was to explore hearing-impaired instrumentalists' perspectives toward HAs and music such that the audiology community can better cater to hearing-impaired instrumentalists' needs.

HAs have largely been developed with speech in mind rather than other complex auditory information such as music. This is intuitive as hearing-impaired listeners' first complaint most often relates to speech understanding. Outcome assessment tools, such as the speech intelligibility index, and HA signal processing mechanics, such as wide dynamic range compression (WDRC), frequency lowering, adaptive noise reduction (ANR), and feedback cancellation, have primarily been developed to improve speech understanding. For example, the speech intelligibility index is a metric used during HA fittings, which predicts speech intelligibility through an HA using weighted speech-frequency regions that are audible to the wearer (Amlani et al, 2002). WDRC compresses the speech output dynamic range by providing more gain for quieter sounds and less gain for louder sounds. Frequency lowering is an additional signal processing mechanism that targets high-frequency syllabic information and lowers it to within the audible bandwidth for the listener (Alexander, 2013). Another additional feature, ANR, relies on detecting acoustic modulations typical of speech to suppress the relative level of background noise that may be interfering with the speech signal (Bentler and Chiou,
2006). Each of these features has been designed to enhance speech understanding. However, because of the differences in acoustic properties between speech and music (Chasin and Hockley, 2014), it is possible that these same features can have an adverse effect on the perception of music.

Several surveys have addressed hearing-impaired listeners' music-related complaints by questioning respondents about HAs and music. Feldmann and Kumpf (1988) relate that $79 \%$ of their survey respondents reported that their hearing impairment interfered with music enjoyment, with complaints relating to understanding lyrics as well as pitch and melodic distortions. About two thirds of the respondents reported that HAs improved music listening, but that they still struggled to perceive rapid sound level changes. Leek et al (2008) found that almost $30 \%$ of their respondents were dissatisfied with music listening, attributing the largest challenges to sound level issues, and the authors attributed the reduction in complaints to advancements in HA technology over the two decades between the studies. Most recently, Madsen and Moore (2014) conducted a survey which specifically identified issues that HA users encountered listening to music. Overall, the most prominent problems identified were distortion, feedback, inappropriate gain, unbalanced frequency responses, and reduced tone quality.

In current hearing aid fittings for music listening, clinicians are encouraged to disable the HA signal processing mechanics described previously when fitting HAs for music listening (Moore, 2016; Zakis, 2016). Whether these signal processing adjustments are applied in practice and if they are associated with improved music listening experiences remain unknown. More importantly, and underscoring the need for this current research, is that these surveys only address individuals' experiences listening to music and not instrumentalists' experiences participating in and performing music.

The challenge when using surveys to understand listeners' needs is linked to music's holistic nature. Improving music is much more than removing negative auditory descriptors such as distortion, feedback, and reduced tone quality. Indeed, Small (1988), in his groundbreaking book, Musicking, debunks music as an object in that the "fundamental nature and meaning 
of music lie not in objects, not in musical works at all, but in action, in what people do" (p. 9). In the context of this research, then, music is not simply the act of listening. Rather, music exists between and within the participatory relationships that are produced with sounds and others. Indeed, music can produce intrinsic enjoyment, emotional rewards and social fulfillment, among other benefits (Coffman and Adamek, 1999; Fulford et al, 2011). Although these benefits may contribute to and enhance instrumentalists' experiences listening, responding to, and performing music, these benefits may not necessarily be contingent on removing undesirable auditory descriptors. Bartel et al (2011) conducted a qualitative case study on cochlear implant users and music appreciation. One of their participants reported high enjoyment of music despite poor self-reports of auditory abilities, including poor rhythm, tone, and timbre perception. Thus, although questions relating to music's auditory nature can shed light on the degradation of auditory perception due to hearing loss, these same questions might not relate to a listener's ability to achieve some of music's holistic benefits. James Strachan, former chief executive of the UK's Action on Hearing Loss charity and HA user, succinctly articulated the issue:

\footnotetext{
Hearing speech is a binary phenomenon: either you understand, or you do not. Whereas appreciating or enjoying music is a range phenomenon: just as I do not know how you see the color red, I do not know exactly what you hear when you listen to Adele or Beethoven (Strachan, 2016).
}

This anecdote beautifully underscores the ways in which music is a complex and multifaceted concept that cannot be fully understood on the basis of direct auditory questions.

Other challenges and perspectives relating to music's holistic nature may not be accessible through quantitative surveys and are better revealed through the use of qualitative methods. Fulford et al (2011) conducted semistructured interviews with hearing-impaired musicians to determine the ways in which their musical experiences were impacted by hearing loss. In their sample, music self-efficacy was motivated by family encouragement from an early age, regardless of hearing status. Furthermore, participants reported a variety of listening styles, including reliance on auditory cues and other sensory and attentional cues as well. These findings point to fulfilling music listening strategies that operated independently of challenges due directly to hearing impairment. Fulford et al (2012) revisited their 2011 interviews to screen for reports related specifically to HAs. Several of the participants were dissatisfied with modern digital HAs and had complaints of distortion which lead to some participants rejecting HAs altogether. Other participants reported that HAs were simply able to give them access to music, using strategies such as an HA music program or adapting to their technology over time. Not only do these studies reveal that qualitative methods produce findings that are consistent with quantitative findings but they also suggest that qualitative research methods reveal ways in which hearing-impaired musicians enjoy fulfilling musical experiences beyond auditory descriptors, which may not have been identified using quantitative methods. What remains to be known is the relationship between auditory impairments and fulfilling musical experiences and whether one impacts the other.

The purpose of the present study was to explore the challenges that adult HA-wearing instrumentalists face that prevent them from listening, responding to, and performing music. Our broadly conceived research question was how do adult instrumentalists report the impact of hearing impairment and HA use on music listening, responding, and performing, and on social participation in an instrumental setting? To that end, we employed conventional content analysis as a research method (Hsieh and Shannon, 2005) to analyze short oral histories collected from adult amateur instrumentalists participating in a local community wind band organization. The goal was thus to aggregate their experiences to help explore prominent auditory deficiencies and challenges which inhibit musical participation that might be ameliorated through the use of HA technology and rehabilitation. Given the relative lack of literature reporting positive musical outcomes compared with positive speech outcomes following HA intervention, we chose to frame our research question around the challenges of musical participation so that our findings could set the stage for others to develop targeted rehabilitation strategies for music-based concerns.

\section{STUDY DESIGN}

$T$ he researchers conducted semistructured interviews with adult amateur instrumentalists to gain better insight into their experiences and perspectives related to the research question. Before addressing the design in more detail, descriptions as to who we are as researchers are necessary to frame the positionality and reflexivity found throughout this project. Authors J.V. and P.F. have extensive experience in quantitative audiology research methods and relatively less experience conducting qualitative methodologies. P.F. has an interest in music personally and professionally as it relates to HA user satisfaction and digital signal processing challenges for those with hearing loss. J.V. has a background as an instrumentalist but studied formally only through high school. Author J.V. also has a strong background in music cognition theories and research methods from his undergraduate education. 
Author A.M. has more experience in qualitative audiology research methods than author J.V. Although author C.B. has little to no experience of audiology, her experience in qualitative research methods and her background in instrumental music performance and music education brought another level of expertise to the team. The diversity of this research team is such that the strength of the study only benefited from the multiple perspectives held between the four authors.

During the interviews, each researcher wrote memos that were referred to throughout the research process by each of the other researchers. Once the interviews were transcribed, conventional content analysis was used to describe, analyze, and synthesize categories that emerged from interview transcripts, personal memos, and collective insights. Through the analysis, key thoughts and/or concepts (Hsieh and Shannon, 2005 , p. 1279) emerged as findings, and categories were drawn from the data. A multidisciplinary perspective between the researchers helped identify the emergent codes and categories (Hsieh and Shannon, 2005). Given the authors' experiences and the literature reviewed in this study, it was recognized that hearing loss and HA use has the potential to negatively impact music perception and performance. During the interviews, the authors generated dialogue using semistructured questions and then probed areas of interest. This allowed the construction of categories that emerged as a result of interactions between the authors, the field, and the participants.

\section{Sampling}

Purposeful sampling was employed to yield "informationrich" (Patton, 2002) data from knowledgeable participants. The study participants included in the final data analysis were selected because they had experiences that would "purposefully inform an understanding of the research" (Creswell, 2007, p. 125). That is, participants with unique experiences of the phenomenon studied were sought so that the findings could arise from the data generated from the sample studied (Knudsen et al, 2012). Therefore, the authors aimed to recruit experienced hearing-impaired instrumentalists who could confidently articulate their perceptions of music listening and performance.

Participants were recruited from several sources. Initially, the study was advertised to an amateur ensemble band consisting primarily of older adults in London, ON, Canada. Most of the participants were retirees. Based on the statistics collected by the Canadian Health Measures Survey, it was anticipated that a significant portion of these individuals would exhibit some degree of hearing loss due to age (Feder et al, 2015). Participants with known musical experience were also recruited from the National Centre for Audiology patient database, as were personal colleagues of the authors who fit the preliminary criteria.

\section{Participants}

A total of 54 participants were recruited and interviewed. For all participants, a detailed case history, pure-tone audiometric thresholds $(0.25,0.5,1,2,3,4$, 6 , and $8 \mathrm{kHz}$ ), word recognition scores at a comfortable listening level, speech recognition thresholds, and tympanometric measurements were collected. One participant's interview was conducted via voice-overinternet-protocol (VoIP), and his most recent hearing assessment was faxed from his local audiologist. Among the 54 participants recruited, 49 presented with some degree of hearing loss (a threshold above $25 \mathrm{~dB} \mathrm{HL}$ for at least one frequency). Among the 49 participants with hearing loss, 24 presented with a three-frequency puretone average threshold (PTA3) across $0.5,1$, and $2 \mathrm{kHz}$ $>25 \mathrm{~dB}$ HL in at least one ear. There were a total of 15 HA users, 14 of whom had a PTA3 $>25 \mathrm{~dB}$ HL in at least one ear and one whose PTA3 was $<25 \mathrm{~dB}$ HL in both ears.

After a brief initial review of the interview transcripts, it was clear that not all of the participants had sufficient musical experience to confidently articulate the phenomenon in question. The adult music group, from which most of the participants had been recruited, turned out to be for many a place to begin music study at the most basic level. This meant they were engaging in the formal study of music for the first time in their lives: learning how to play a musical instrument; how to read music; and how to respond to the conductor and other instrumentalists around them. In addition, a portion of the individuals among the 49 participants with hearing loss had minimal high-frequency hearing loss. Some of these individuals did not find their hearing loss to impact their day-to-day lives, and as a result did not seek intervention. Therefore, the authors selected a subset of participants whom they believed to have sufficient hearing loss and musical experience for the purposes of this study. Their characteristics are described in the following paragraphs.

A total of twelve participants' (seven male, five female) interview transcripts were analyzed for this study (author C.B. is using the full dataset $[n=54]$ to explore the degree to which amateur musicians [normal hearing or hearing-impaired] focus their listening attention on the conductor rather than the sound of the ensemble and the associated musical education implications in a separate report). Participants' ages ranged from 55 to 83 years $($ mean $=67.8, \mathrm{SD}=9.5)$ and each participant exhibited some degree of hearing loss. Most of the cases were sensorineural hearing loss ranging from mild to severe. There was one instance of moderately severe mixed hearing loss with a mild conductive component. All participants wore HAs and had between 
two and 43 years of HA experience $($ mean $=18.9, \mathrm{SD}=$ 16.0). In addition, all participants had at least four years of musical experience, with the majority exhibiting 40 or more years (mean $=32.7, \mathrm{SD}=19.9$ ). Musical experience was defined as taking private instrumental lessons, experience performing in an instrumental ensemble, writing, arranging and producing musical content, or a combination of all of these. A detailed breakdown of the twelve participants' hearing characteristics can be found in Table 1. All participants were financially compensated for their participation in this study (including those whose interviews were not analyzed). This study was approved by the Western University Health Research Ethics Board.

\section{Data Collection}

The semistructured interviews with the participants ranged in length from 20 to 60 minutes and were completed in a single session in a quiet laboratory at the National Centre for Audiology either in person or via VoIP. To minimize researcher bias and to maximize interviewer sensitivity, authors J.V., P.F., and C.B. took part in developing the interview guidelines. Together, they developed an interview guide, loosely adapted from Leek et al's (2008) telephone survey questionnaire investigating hearing-impaired listeners' enjoyment of music. The interview guide was designed to encourage discussion topics ranging from when the participant identified their hearing loss, how long they have participated in music ensembles, whether sound quality was affected, and the effectiveness of their HAs. From the beginning of the study, the researchers were aware of the need to situate biases and beliefs even in the development of the interview questions (Berger, 2015). Although semistructured interviews permitted the use of open-ended questions, there was also the need to provide prompts so that participants could provide and elaborate on accounts of their experiences of the phenomena under study (Knudsen et al, 2012). Two of the researchers had experience as performing instrumentalists and two were experienced in audiology procedures; thus, we were constantly cognizant of our abilities to both promote and perhaps hinder on-going dialogue between the interviewers and participants. After discussion and reflection between the researchers, the topics of interest that emerged included HA listening habits, HA program use, music components such as timbre, dynamics, and melodic recognition, and social participation goals and needs. The interview guide was piloted with several participants to verify that it effectively promoted dialogue, encouraged meaningful contributions based on each author's background, and allowed for descriptions to emerge. An example of interview questions can be found in Table 2.

Interviews were conducted by authors J.V., P.F., and C.B. Participants chose interview times that were convenient for them. Many of the participants never had a hearing assessment or visited an audiology laboratory. Recognizing that we could not predict how participants would respond to perceived social, professional, and educational positioning (Berger, 2015; FinefterRosenbluh, 2017), great care was taken to make the interview as comfortable as possible. Thus, the participants were greeted by one of the authors in the reception area, walked through the building to the lab, and then offered a beverage as they settled in. As described earlier, each participant then provided a detailed case history and completed an audiological assessment before their respective interview.

The data consisted of transcripts and memos collected by the interviewers during the interviews. Interviews in person were recorded using Audacity (version 2.0.6) software using a built-in laptop microphone, and interviews via VoIP were recorded using Skype (version

Table 1. Participant Characteristics

\begin{tabular}{|c|c|c|c|c|c|c|c|c|c|c|c|c|c|c|c|c|c|c|c|c|c|c|c|}
\hline & \multirow[b]{2}{*}{ Gender } & \multirow[b]{2}{*}{ Age } & \multirow[b]{2}{*}{ ME } & \multirow[b]{2}{*}{ HAE } & \multirow[b]{2}{*}{$\mathrm{HLT}$} & \multirow[b]{2}{*}{ HAS } & \multirow[b]{2}{*}{$\overline{B M}$} & \multicolumn{8}{|c|}{ Left Ear, Frequency $(\mathrm{kHz})$} & \multicolumn{8}{|c|}{ Right Ear, Frequency (kHz) } \\
\hline & & & & & & & & 0.25 & 0.5 & 1 & 2 & 3 & 4 & 6 & 8 & 0.25 & 0.5 & 1 & 2 & 3 & 4 & 6 & 8 \\
\hline$\overline{\mathrm{P} 1}$ & $\mathrm{M}$ & 83.4 & 4 & 25 & SN & BTE & $B$ & 60 & 65 & 75 & 75 & 80 & 85 & 85 & NR & 55 & 50 & 45 & 55 & 65 & 65 & 75 & 80 \\
\hline P2 & $\mathrm{F}$ & 60.4 & $>40$ & 15 & SN & $\mathrm{ClC}$ & B & 50 & 55 & 60 & 60 & 60 & 60 & 65 & 75 & 70 & 60 & 65 & 55 & 60 & 65 & 65 & 80 \\
\hline P3 & $\mathrm{F}$ & 58.8 & $>40$ & 2 & SN & $\mathrm{RIC}$ & B & 10 & 15 & 20 & 45 & 50 & 45 & 30 & 20 & 0 & 5 & 15 & 40 & 50 & 45 & 30 & 20 \\
\hline P4 & $M$ & 75.5 & $>50$ & 3.5 & SN & ITE & B & 20 & 20 & 25 & 70 & 65 & 70 & 70 & 75 & 20 & 20 & 20 & 60 & 70 & 70 & 75 & NR \\
\hline P5 & $M$ & 76.2 & 15 & 12 & SN & $\mathrm{RIC}$ & B & 30 & 35 & 50 & 50 & 55 & 80 & 105 & NR & 35 & 35 & 50 & 45 & 50 & 75 & 90 & NR \\
\hline P6 & $\mathrm{F}$ & 75.8 & 5 & 6 & SN & RIC & B & 30 & 25 & 40 & 45 & 50 & 50 & 60 & 75 & 20 & 30 & 45 & 55 & 50 & 50 & 45 & 70 \\
\hline P7 & $F$ & 59.2 & $>50$ & 4 & SN & ITC & B & 15 & 15 & 10 & 30 & 35 & 30 & 40 & 55 & 30 & 20 & 20 & 30 & 30 & 25 & 45 & 45 \\
\hline P8 & $M$ & 72.1 & 40 & 42 & SN & $\mathrm{RIC}$ & B & 50 & 45 & 45 & 65 & 60 & 65 & 75 & 80 & 25 & 25 & 35 & 55 & 65 & 75 & 85 & 85 \\
\hline P9 & $M$ & 74.4 & 5 & 4.5 & SN & $\mathrm{RIC}$ & B & 25 & 30 & 20 & 30 & 45 & 50 & 55 & 55 & 35 & 30 & 25 & 35 & 55 & 60 & 60 & 65 \\
\hline P10 & $\mathrm{F}$ & 56.4 & 42 & 31.5 & SN & BTE & B & 70 & 75 & 70 & 60 & 60 & 60 & 55 & 65 & 80 & 80 & 75 & 65 & 65 & 65 & 65 & 65 \\
\hline P11 & $M$ & 55.8 & 40 & 43 & SN & ITC & B & 45 & 55 & 65 & 75 & 85 & 85 & 80 & 75 & 50 & 55 & 65 & 65 & 75 & 75 & 95 & 80 \\
\hline P12 & $M$ & 65.5 & 61 & 37.5 & $M$ & BTE & B & 75 & 70 & 75 & 65 & 55 & 60 & 65 & 65 & 75 & 85 & 80 & 75 & 55 & 55 & 65 & 50 \\
\hline
\end{tabular}

Note: Gender (M = male, F = female), age (years), music experience/ME (years), hearing aid experience/HAE (years), hearing loss type/HLT ( $S N=$ sensorineural, $M=$ mixed), hearing aid style/HAS (BTE = behind-the-ear, CIC = completely-in-the-canal, RIC = receiver-in-the-canal, $\mathrm{ITE}=$ in-the-ear), binaural or monaural/BM $(\mathrm{B}=$ binaural, $\mathrm{M}=$ monoaural), audiometric data ( $\mathrm{dB} \mathrm{HL}, \mathrm{NR}=$ no response). 


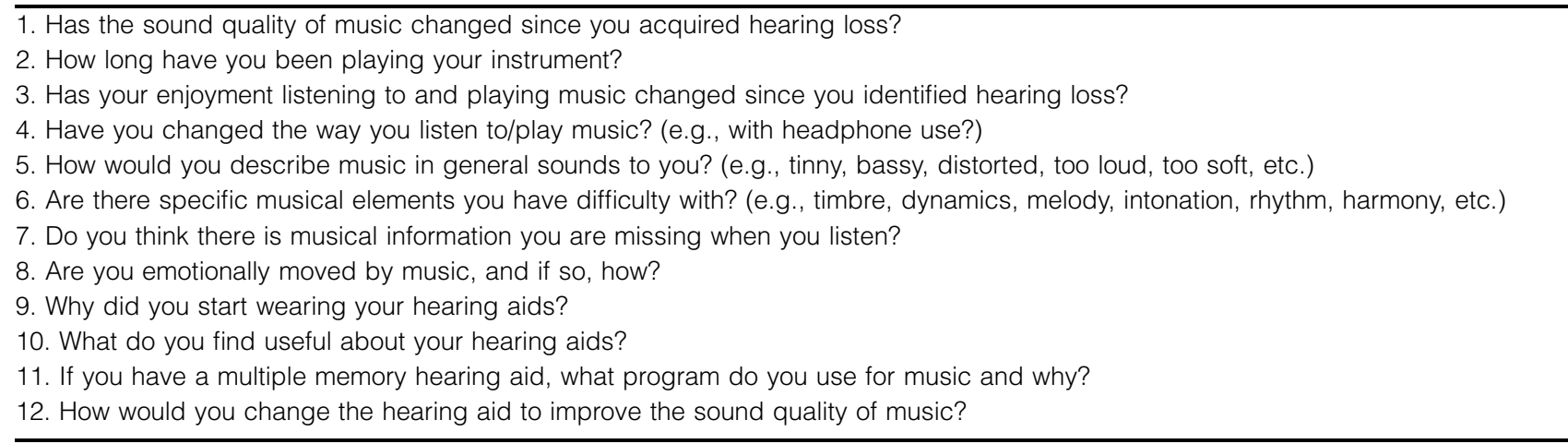

7.37) software. All files were converted to MP3 format and confidentially transcribed verbatim by a thirdparty transcriptionist. The authors then verified that the transcripts' contents were consistent with the memos that had facilitated the tracking of ideas and concepts throughout the duration of the research study. Although it was important to verify consistency between the interview transcripts, the interviewers' impressions of the transcripts, and the final report (Van Den Hoonard, 2012), the researchers were keenly aware of possible bias. To minimize bias, the researchers, throughout the process, regularly met to cross-check their responses to what was read in the transcripts, what was remembered in the moment of the interviews, and the notes taken in the form of memos.

\section{Analysis}

The authors analyzed the data using conventional qualitative content analysis, as outlined by Hsieh and Shannon (2005). First individually, and then throughout multiple meetings, the researchers thoroughly coded the data for emerging themes that spoke to each of us. However, authors J.V. and C.B. had a working "start list" (Miles and Huberman, 1994, p. 58) of themes they suspected might emerge based on their diverse personal, professional, and disciplinary backgrounds. Because author A.M. had less background as an instrumentalist, she did not begin with a "start list." Rather, she read for themes using her background as a qualitative audiology researcher and checked for consistency with the data analyzed by authors J.V. and C.B.

After reflection and discussion, we determined a set of themes on which we were all in agreement. Once this set of themes was decided on, the authors continued reading for other instances which could be coded into the same themes. Once trending ideas among the codes emerged, the authors formed categories, or broader ideas representing a grouping of codes, consisting of multiple participants' perspectives (Miles and Huberman, 1994). The coding process was repeated for each category. Throughout this process, the researchers were also describing and framing the categories in support of the research question. To exemplify: One participant reported, "Well I think you're going to have more trouble hearing music that's very quiet, for sure." This was coded as "difficulty with very quiet sound," which was subsequently grouped into the category "dynamics" as part of "affected musical components." Another participant commented, "When I was playing [with HAs], certain notes I would hit and I would get feedback." This was coded as "certain musical notes create feedback," and was eventually grouped into the "HA sound quality" category. Both these categories represent challenges that hearing-impaired instrumentalists encounter.

The authors aimed to maintain trustworthiness throughout the analysis. Trustworthiness in qualitative research has been considered analogous to validity and reliability in quantitative research (Golafshani, 2003). Trustworthiness consists of multiple components such as credibility, transferability, and dependability (Guba, 1981; Shenton, 2004; Knudsen et al, 2012; Sikolia et al, 2013). Credibility was achieved by coding data from various sources: interview transcripts, memos, case histories, and the authors' impressions. Dependability was achieved in the present study by reflecting on and discussing emerging categories at each phase of the coding process. Although transferability is not a stated goal of qualitative research, it can occur when some or all of the study findings can be transferred to another similar context (Guba, 1981). We are hopeful that transferability of this study can be achieved because of the trustworthiness of the description of the study, the presentation of the data, and anticipated consistency of the data with other research studies. We also anticipate that our findings relating to instrumentalists and their experiences with hearing loss could likely be transferred to some degree when studying other kinds of instrumentalists, possibly within other cultural practices, in other geographical areas, and/or of varying gender and age who also have hearing loss. 


\section{Reflexivity}

As a qualitative methodology was deemed the most appropriate for this study, it is necessary to recognize the issue of subjectivity in the research process. Reflexivity refers to an awareness of subjectivity, or more specifically, of how the authors' own presence influenced the research process (Barry et al, 1999) and is considered an essential component in qualitative research (Watt, 2007). Historically, research in the sciences aimed to rid elements of bias and subjectivity from research designs (Wilkinson, 1988). This stance, however, has been challenged by social psychologists, feminist theorists, and critical race scholars (Gough and Madill, 2012 , p. 374). In this study, the authors not only embraced the strengths and possibilities embedded in intersubjectivity but also understood the impossibility and falseness of claiming a completely objective stance. In this study, the authors' subjectivity, in essence, was "reviewed as a resource that [was] tapped in order to contextualize and enrich the research process and its products" (p. 375). Thus, the authors sought throughout the research process, and now here in this article, to make explicit their "understanding, positions, and approaches" (Gentles et al, 2014, p. 3) towards their interactions with the participants and amongst themselves, the influence each may have had on the other, and the distinct influence each brought to how the data were viewed.

Throughout the study, as has been previously articulated, the researchers met at various times during and after data collection to think out loud as to their reactions and perceptions of the engagements of the participants and other issues found in our memos and transcripts. During meetings, the researchers discussed personal memos, perceptions of the data, and interview transcripts and looked for intersections and commonalities among them. Perhaps, seemingly peripheral to this study are the conversations we had that on focused interdisciplinary connections based on valuing and values that were distinct to our disciplines. The kinds of questions we were interested in pursuing became one of the more powerful focal points as we became aware of our internal reflexivity and we became more comfortable with embracing and sharing that which we thought we knew and that which we came to find we did not. Although this study is not categorically labeled "interdisciplinary," we do hail from different disciplines; thus, the recognition of the kind of language each of us used and our epistemological stances, not just in the interviews but with each other, helped to keep this study rigorous. Indeed, personal, professional, and disciplinary reflexivity (Wilkinson, 1988) was not only present throughout but also facilitated the entire arc of the research process, as well as our own growth and transformation as scholars, researchers, and practitioners.

\section{FINDINGS}

$\mathrm{E}$ ven to those not grounded in audiology research or the performing arts, it is not surprising to state that one of the central findings that emerged was that hearing-impaired instrumentalists encounter challenges participating in their music activities. Three categories (themes) emerged that help exemplify the findings. The first and most prominent category consisted of the participants' participatory needs; hearing loss mostly interfered with their ability to hear the conductor, which they believed to be necessary to participate during rehearsals. The second category consisted of the participants' impressions about HAs: sound quality was influenced by HAs and satisfaction using an HA music program. The third category consisted of the effects of hearing impairment on music perception and included the following subcategories: missing auditory information, affected music components, and nonauditory music perception strategies.

\section{Participatory Needs: Hearing the Conductor}

In his research with adult musicians in a community organization, Jutras (2011) categorizes the benefits of participating in an adult New Horizons Band in Rome, Georgia, finding that skill-related and social/cultural were the two most frequently identified reasons for participation. These findings resonate with this study in that participation did comprise "social interaction, social relationships, and socialization" (p. 67) and skill development, including "skill improvement, skill refinement, technique, musicianship, music theory, music listening, and musical knowledge" (p. 67).

However, our research differs from the study of Jutras in that teasing out reasons for participation was peripheral to our goal of understanding from the adult instrumentalists' perspective what it was like to listen, respond to, and perform music. Participation was given as each of the participants was actively playing in an ensemble(s) of some kind. Thus, one assumption going into this study was that these instrumentalists' motivation to wear HAs would be to better hear and discriminate their music making. However, one of the more prominent difficulties reported by participants in an ensemble was being able to hear the conductor's directions during rehearsal. This was the primary reason that participants chose to wear their HAs.

"One of the reasons I started to get HAs was so I could just hear [the conductor] while playing." 
"I had to keep my HAs in so I can hear [the conductor], like, when she's making comments and things like that."

Some participants reported that hearing the conductor was the only challenge related to their hearing loss and HA use, and that they did not actually experience any difficulties perceiving music itself. The following are examples of these sentiments.

"Without the HAs, I can't really say, because I have to have my HAs in, you know, in order to, you know, hear the conductor."

"And then my concern was well, should I wear them when I'm the band, will it be too noisy? But then you've got to hear what the conductor's saying, so I wear them now."

Thus, interestingly, rather than using HAs to hear what is happening musically so that they may respond musically, listeners' primary motivation to wear HAs during rehearsal appears to be listening to and understanding the conductor's instructions. For some, this was the only motivation to wear HAs.

\section{Participants' Impressions of Hearing Aids}

Despite the need to understand the conductor, the use of HAs would inherently have some sort of impact on the acoustic content of music processed by HAs. Two subcategories emerged related to impressions of HAs and their effect on music. The first concerned how HAs affected sound quality of music. The second related to the use of an HA music program. Some participants briefly commented on what they believed would improve music listening through HAs, although this was not grouped into a separate subcategory.

The participants expressed highly variable opinions regarding the impact of HAs on music sound quality. Some participants expressed positive views, "When I have the HAs in, the clarinet is louder...it seems brighter and sharper than without." Other participants expressed negative views, "I found that I got the real quality, you know, the real actual feel of the music without my HAs." Some participants also had neutral opinions, "I don't think the HAs make a lot of difference." These examples portray considerable variability in satisfaction of HA-amplified music across individuals.

One participant expressed that previous analog HAs provided better music sound quality compared with more recent digital HAs because the digital aids limited the amount of acoustic information that was amplified: "My best sounding set of HAs ever were analog. They had no bells or whistles. It was just straight gain and these had a very extended range. I had a big problem with digital aids...because of the hard cap." This anecdote associates fidelity of sound quality with signal processing schemes found in analog HAs, compared with digital HAs, and could, with more research, direct researchers to replicate analog processing strategies in modern digital systems.

Participants were further asked if they had experience using an HA music program (an HA music program is a set of HA-processing parameters adjusted with the aim of optimizing a music signal, although the exact adjustments vary across manufacturers) to improve music sound quality. Some participants reported no benefits when using a music program, "the music settings. . just don't provide any benefit to me, I don't see any difference." Other participants reported never using a music program. Some participants found a music program to be helpful because it improved the balance and brightness of sounds. However, one of them mentioned that the effort to change the HA setting was not worth sacrificing the convenience of leaving their HA at one setting, "I think [a music program] improves [music listening], but for me it's...with these HAs it's just easier to leave it set at one thing." These statements suggest that music programs are ineffective at improving music sound quality relative to a typical HA program, and even when they are, the relative improvement in sound quality is not worth the effort to change the HA settings.

When asked what it would take to build a better HA, most participants were unsure what would improve music sound quality. However, there were a few characteristics mentioned. One participant suggested that a wider frequency bandwidth would improve the response. This is a reasonable suggestion as most HAs amplify only between $200 \mathrm{~Hz}$ and $6 \mathrm{kHz}$, despite optimal speech and music sound quality being associated with wider bandwidth in both high and low frequencies (Moore and Tan, 2003). Other participants suggested that background noise could be lowered relative to the signal of interest. This suggestion is consistent with current technologies as ANR systems are capable of lowering the background noise level without noticeable speech sound quality degradations (Bentler et al, 2008; Scollie et al, 2016). It is worth investigating the effect of ANR systems on music stimuli. In addition, another participant wished that loud and soft sounds could be more effectively balanced. This last suggestion may be interpreted as a dynamic range issue. This is no surprise, as HAs are typically built for the dynamic range of speech, although the dynamic range of live music is much greater (Chasin and Russo, 2004). Together, these findings show that most listeners do not consider strategies to improve the sound quality of music using their HAs. However, those who do consider strategies share insights that are consistent with evidence found in the literature. 


\section{Effect of Hearing Loss on Music Perception}

Although the use of HAs certainly impacted music sound quality, we were also interested in how HAs and hearing impairment impacted specific aspects of music. We therefore asked participants more targeted questions in these areas. The participants' responses were grouped into three subcategories: awareness of missing information, affected musical components, and multisensory music perception.

When asked if something about a musical signal was affected or missing, many of the participants responded that they thought something was missing. However, they were unable to identify exactly what it was. In fact, some participants reported being unsure what the music was exactly supposed to sound like: “... how do I know what it should sound like? So I just listen to it according to my hearing deficiency, whatever, not knowing what the real thing might be." Another participant said, "with my hearing, and with wearing HAs, you don't know what you're missing." Some participants described that they did not attempt to identify missing sounds until it was brought up in the interview. When asked about what instruments they may not hear, one participant expressed, “...it's one of those things where I haven't sat down and tried to put my finger on." Some participants reported that they have had hearing loss for so long that they could not remember what "normal" music sounded like. Their sense of normal had implicitly changed and they were not able to describe how music "should" sound. These reports suggest that hearing-impaired listeners generally suspect they are missing musical information. However, they do not consider missing musical content to be a significant concern, nor do they find it a barrier to musical participation.

To resolve what musical information might have been missing, participants were probed about specific music components. The components most frequently discussed during the interviews were dynamics, intonation, melody, and timbre. During the interviews, the authors defined dynamics as the relative contrasts of loud and soft levels of music. Intonation was defined as the realization of pitch and whether or not the pitch is in tune. Melody was defined as the principal succession of pitches in a musical composition. Timbre was defined as the characteristics of the sound which allowed the listener to identify what instrument is playing.

Many participants expressed concerns with musical dynamics as a result of hearing loss, HA use, or both. They described having difficulty identifying and contrasting loud and soft occurrences. Some participants expressed difficulty perceiving and playing soft music relative to loud music: "...you're going to have more trouble hearing music that's very quiet." In addition, another participant mentioned: "the softer instru- ments ... [are] probably harder for me to hear." Other participants suggested that dynamics were easier to perceive without HAs: "in fact it's probably more accurate without my HAs." These reports suggest that hearing loss and HA use may make differentiating and performing loud and soft contrasts in music passages more challenging.

With respect to intonation, several participants reported positive experiences staying in tune despite their hearing loss and HA use once they were proficient at their instrument. For example, one participant reported, "I'm rarely very far out of tune." However, another participant suspected that difficulties with intonation are not crucial for overall music perception, "as far as hearing something in tune or out of tune...I'm not sure those are crucial things." These reports suggest that intonation is an aspect that amateur musicians may not associate with challenges due to hearing impairment and HA use, especially with sufficient training on their chosen instrument.

Melodic recognition was particularly challenging for almost half the participants. They expressed that it was difficult to identify the melody if there was too much noise, if there were too many instrumental parts, or if the melody was playing particularly quietly. One participant expressed difficulty following the melody "especially when it's a softer sound." Another said, "I can't do it if they're all played at the same time, it just sounds like mush." This is consistent with previous surveys in which HA users struggled to listen to musical lines in layered ensembles relative to solo instruments (Leek et al, 2008; Madsen and Moore, 2014). These findings suggest that melodic recognition can be affected by other musical parts and noise generated in a rehearsal space. Background noise, whether it is related noise or musical layering, appears to worsen participants' abilities to recognize melodies. This recognition challenge may be interpreted as being analogous to difficulties understanding speech in noise for hearing-impaired listeners.

Finally, many participants articulated positive experiences related to instrumental timbre discrimination and identification. When asked if they could discriminate and identify instrumental timbres, many participants were confident that they could: "I could pick out the oboe from the clarinets [when listening to a performance]." Participants also reported various acoustic cues that helped them identify different timbres. Such cues included the register in which the instrument plays, the intensity that the instrument typically produces, and the quality of sound. For example, one participant said, "I can hear [the piccolo], but that's just because you're an octave higher than anyone." Difficulties attributed to instrumental timbre perception were related to these cues. Some participants reported difficulty discriminating instruments if they played in a 
similar register or at a similar level. These findings suggest that hearing-impaired listeners easily discriminate various timbres and rely on acoustic cues to do so. Although timbre discrimination and identification deficits have been identified in listeners with moderateto-severe hearing losses (Emiroglu and Kollmeier, 2008; Looi et al, 2008; Uys and van Dijk, 2011), deficits have been more variable between individual hearingimpaired listeners exhibiting mostly moderate flat losses (Kirchberger and Russo, 2015). Given that most of the participants in the present study presented with moderate flat hearing losses, these previous quantitative findings are in agreement with some reports identified here.

Given the impact of hearing impairment and HA use on music perception, we asked participants about using nonauditory senses to supplement the musical experience. Some participants expressed that they believed their somatosensory system could supplement their auditory system when performing music in an ensemble whether or not they were hearing-impaired or whether or not they were wearing HAs. That is, they could perceive parts of music through touch responses and vibrations against and within their body to enjoy music and to monitor musical intonation. With respect to enjoying music, one participant mentioned, "in a hearing-impaired person, feeling is just as valid a method of hearing as audio perception is." With respect to monitoring musical intonation, a participant said, "I know which pitches on a flute tend to play flat or sharp, so I adjust. . I can feel the vibrations and that's very helpful to me." This is also consistent with the participant previously mentioned who felt that correct intonation comes from "where they feel it."

Although it is not clear if the vibrotactile sensations supplement the perception of some of the musical components mentioned, it is possible that the sensations contribute in some way to music enjoyment beyond the auditory experience. Music has previously been described as a multisensory phenomenon which integrates stimuli from a variety of sensory systems on the basis of cortical evidence in multisensory regions (Zimmerman and Lahav, 2012). The behavioral anecdotes reported here are supportive of the multisensory hypothesis.

\section{Consistency across Other Literature}

Many of the HA-related auditory concerns reported in this study are consistent with findings obtained from both qualitative and quantitative approaches to the effects of hearing impairment and HA use on music perception in other studies. These consistencies support the possible transferability and confirmability of this study's findings, suggesting a trustworthy dataset (Knudsen et al, 2012). Qualitatively, these results were consistent with findings reported by Fulford et al (2011; 2012). In both studies, participants exhibited attitudinal ambivalence in that descriptors about HAs were both positive and negative. Positive descriptors consisted of participants describing HA-amplified music as "brighter" or "crisper." The descriptor "brightness" is considered a positive dimension of sound quality and is associated with a modest increase of the treble portion of the frequency response (Gabrielsson and Sjögren, 1979). Negative feedback consisted of sound quality descriptors that included "screeching" and "tinny." The descriptor "tinny" is a common complaint for HA users and is associated with too much gain in the high frequencies (Jenstad et al, 2003). The descriptor "screeching" may be related to "squealing," which is often used to describe a distortion known as acoustic feedback. Issues of feedback and unbalanced frequency responses have been quantitatively identified as concerns for HA music (Madsen and Moore, 2014). The HA music program's inability to improve music listening also trended across multiple studies. In our study, listeners were generally indifferent about a music program's efficacy. Some participants in Fulford et al (2012) were unsure of whether or not they had a music program, and those who did have a music program did not use it consistently. Madsen and Moore (2014) reported music satisfaction scores that were similar from both users and nonusers of a music program, suggesting that the music program did not significantly affect music sound quality. Vaisberg et al (2017) found that only two of five HA music programs improved music sound quality, and that the magnitude of improvement was less than the variation across HAs. Together, these results may indicate that further improvement in music programs may be desirable. The last consistency between our study and others concerned preferences between analog and digital HAs. One of the current participants preferred legacy analog HAs relative to modern digital aids. This preference was also found among listeners interviewed by Fulford et al (2012). This finding made sense, as analog HAs provide mostly linear amplification. Linear or linear-like amplification has been found to improve music listening compared to the WDRC commonly provided in today's digital HAs (van Buuren et al, 1999; Arehart et al, 2011; Higgins et al, 2012; Croghan et al, 2014; Kirchberger and Russo, 2016).

Many of the auditory concerns due to hearing impairment were also consistent with quantitative literature on similar topics, as discussed previously. However, these concerns also related to the dynamic listening styles reported by Fulford et al (2011). The fact that many of the present study's participants articulated the ways in which their auditory experience was affected highlighted some degree of reliance on hearing for musical participation. However, participants 
frequently discussed supplementing their hearing by using nonauditory attending strategies, such as vibrotactile sensations to perceive musical intonation, as did participants interviewed by Fulford et al (2011). Both auditory and nonauditory listening styles allow listeners to negotiate concerns caused by hearing impairment and distorting effects due to HAs (Fulford et al, 2011) and may, therefore, be considered valid methods of perceiving music.

\section{DISCUSSION}

$\mathrm{T}$ he purpose of the study was to explore the challenges that adult HA-wearing instrumentalists face, which prevent them from listening, responding to, and performing music. The following is a discussion of the findings drawn from the participants' interviews. The categories that emerged are considered as they relate to the research questions and the review of literature. The three main categories were participatory needs, impressions of HAs, and effects of hearing impairment on various aspects of music perception. The most predominant participatory need was connected to hearing the conductor. Impressions of HAs were inferred based on how sound quality was affected by HAs and satisfaction using an HA music program. Aspects of music perception included the subcategories: missing auditory information, affected music components, and nonauditory music perception strategies.

Because of our extensive background in audiology research, we were interested, to some degree, in exploring auditory deficits reported by the participants. However, we were also focused on understanding the challenges that instrumentalists face and the relationship between those challenges and the holistic nature of music perception. Therefore, a qualitative methodology effectively afforded participant the opportunity to share auditory (and participatory) deficits that were important for a holistic music experience. Although the participants certainly did discuss concerns related to their auditory experiences, most of them first expressed listening needs related to their ability to participate in a musical ensemble. This was a notable finding as participants were not directly asked about participatory issues before auditory issues. The fact that participants discussed participatory needs before auditory concerns, without even articulating the connection between them, was an interesting result that was supportive of the purpose of this study.

Participation and participatory needs are not newly expressed phenomena in musical engagements, yet it is an idea that has been too often assumed and taken for granted. More than 25 years ago, Gates (1991) encouraged music scholars to better define participation. He defined what he referred to as a "Typology of Music Participants in Societies" and suggested that participation can be typed as work, serious leisure, and play (p. 16). He underscored that most research to that point had been performed by scholars using "positivist research paradigms and quantitative data gathering strategies" (p. 15) and suggested "[getting] beyond [a] surface level of categorization" (p. 17). Since this 1991 article, numerous studies on participation have been conducted, and for the purposes of this article, we consulted "participation" studies that have taken place with older adults. For instance, Dabback (2008) discussed the importance of structure, health, and well-being that musical organizations provide to adults. He specifically addressed the ways in which music engagement may provide continuance of a musical identity that may have been formed during childhood. He also, however, discovered the importance adult musicians place on the opportunity to reclaim and develop new musical and social identities. Coffman, a researcher in the area of music education for adults, has, for the most part, primarily relied on quantitative methods to address issues based on (among others) intergenerational engagements (Coffman and Levy, 1997), quality of life, well-being and accomplishment (Coffman and Adamek, 1999; Coffman, 2002a), perceived social support (Coffman and Adamek, 2001), meaningful interpersonal relationships (Coffman, 2002b), spirituality (Rohwer and Coffman, 2006), and the experiences of conductors with adult learners (Coffman, 2009). Although the present study, qualitative in nature, did not directly focus on the benefits of participation, it was the major category that emerged from the data. It is perhaps through discussing participation that the participants interviewed tried to express some of these benefits embodying the holistic nature of music.

Participating in musical contexts does require some degree of musical understanding, skills, and auditory awareness. One expectation then would be the necessity to hear the music that is being produced around you, whether that means listening for a melody line to balance and tune your own playing or attending to the members in your instrument section to play in tune as a section. Interestingly, however, being able to hear other sections of instruments, the melody, or even the person playing next to you did not emerge as a need, and thus, a category. When participants were asked whether they ever played without wearing their HAs, almost all of them admitted to doing so at one time or another, even during concerts. This suggests that participating in a musical context is not always contingent on rehabilitation of auditory deficits.

Although not all of the participants interviewed were dissatisfied with their HAs, some articulated negative concerns regarding HA sound quality and music programs. These findings are consistent with previous quantitative surveys where many HA users were dissatisfied with music sound quality (Feldmann and 
Kumpf, 1988; Leek et al, 2008; Fulford et al, 2012; Madsen and Moore, 2014). However, the participants in the present study expressed similar concerns to previous studies only after first discussing participatory needs, further highlighting that there are aspects in the holistic music experience that should be rehabilitated before restoring negative auditory deficiencies. What is still not yet fully understood is the relationship between auditory deficiencies and music participation and the degree to which HA dissatisfaction inhibited the instrumentalists from playing or listening to music.

\section{LIMITATIONS}

$\mathrm{T}$ his study only included individuals with hearing loss who were active instrumentalists. These findings do not reflect the experiences of hearing-impaired instrumentalists who are no longer musically active. There may be individuals so affected by hearing loss or so disappointed with HA sound quality that they are unable to participate in a music ensemble and have chosen to leave musical ensembles. The recruitment strategy administered here did not allow for the inclusion of such a population. Some participants said that past members may have left the adult music group because of hearing loss. Future studies with a similar methodology should pursue recruitment strategies that allow the inclusion of instrumentalists who, as a result of their hearing loss, no longer perform music. Future studies can also examine the efficacy of nonauditory attending styles reported by Fulford et al (2011), such as using vibrotactile feedback, and determine if those styles can be advantageous for the rehabilitation of musical participation and if they produce holistic musical anecdotes similar to those expressed by normal hearing instrumentalists. In addition, a revised questionnaire should also include questions focused directly on musicrelated quality of life and participatory benefits, so that the relationship between these topics and auditory concerns due to hearing loss and HA use can be better articulated.

Unfortunately, most of the 49 participants with hearing impairment interviewed in this study were not HA users or were not able to articulate their musical experiences in a way that the researchers perceived was informative. This limited useable data to that of only twelve of the participants. Future recruitment strategies should target a larger population of HA users who are instrumentalists but also have experience in areas such as acoustics and hearing science. This may allow a more informative articulation of auditory deficiencies. Future studies may also wish to incorporate sessions in which hearing-impaired participants listen to and perform music and then reflect on their experiences immediately after the session during an interview. This would allow personal accounts of recent musical experiences, richer descriptions of data, and even the inclusion of additional participants.

It will also be valuable to reenter the data pool and consider the music education implications emerging out of the findings. For instance, several of the findings of this study, such as carefully listening to those around you (intonation), being able to discern melodic and harmonic lines, and the ability to differentiate between instrument timbers, are also challenges for instrumentalists who do not wear HAs. What might the implications be of such findings on how instrumentalists are taught? More revealing than this, however, is that even those who have not gone through the most basic music education program would likely report that when you play an instrument in an ensemble, listening and responding to those around you is integral to the individual agency of musical experience. Clearly, it is important to know where to begin playing so one is in the correct place in the music. A more critical read of the data, however, would also reveal that participating musically in an ensemble is too often based on the conductor making most if not all of the musical and artistic decisions. For further reading to substantiate this point, see O'Toole (1994) who uses Foucault to problematize authority in choral rehearsals. See also Allsup and Benedict (2008) who use a similar critical lens to interrogate the dominance of conductors and their methodological control in wind ensembles.

\section{CONCLUSION}

$\mathrm{I}$ $\mathrm{n}$ the year 2000, Conrad and Gunter wrote the following:

The time is right to break through the conventional boundaries that surround disciplinary inquiry, especially boundaries between disciplines, boundaries separating theory and research from practice, and boundaries separating scholars from practitioners (p. 50).

We came to this study as an interdisciplinary team three years ago after meeting during a weekend seminar that brought multiple disciplines together under the umbrella of Musical Learning Across the Lifespan. We ended up sitting at the same table, thus, in immediate ways, forced to find (or, at the very least, discuss) a common ground. It may have been serendipitous that we ended up together at that table, but we were present at that gathering precisely because we were desirous to break through "conventional boundaries" and craft a way forward that would afford new ways of thinking for each of us.

Like many interdisciplinary teams, we first had to come to terms with assumptions and non-understandings we made about the others' discipline. Issues which 
included the ways in which a review of literature is constructed in our disciplines and favored research paradigms were fascinating and less complex to address. More complex, however, (yet equally as fascinating) were the kinds of questions we had individually been exploring before the present study. For instance, perhaps not completely incomprehensible, but clearly in need of explanation (and one that brought us great joy and laughter), was why one would choose a more pragmatic "what" question above a philosophically grounded "why" question.

In this study, the use of qualitative methodology and conventional content analysis addressed both the what and why questions allowing us to explore the impact of hearing loss and HA use on music perception and participation. The authors discovered that the most common music-related concern for included participants with hearing loss and HAs was not actually related to the perception of music itself-it was related to hearing the conductor to actively participate in music-related activities. Participants' reports of participation were thought to address, at least in part, hearing-impaired instrumentalists' needs, above general auditory complaints. This concern for participation generally took precedent over direct auditory needs. However, many of the auditory concerns reported were consistent with both quantitative and qualitative evidence from the literature. With respect to HA use, some participants reported quality degradations, whereas others reported quality improvements. When probed about improving HAs, participants suggested that an extended bandwidth, improved noise reduction strategies, and a large dynamic range were proposed solutions. With respect to music perception, most participants reported that hearing loss worsens the quality of music. The degradations were mainly attributed to issues in music dynamics and melodic identification.

In conclusion, this study expands a growing body of literature articulating the possible effects of hearing impairment and HA use on music perception and highlights what may be important for amateur instrumentalists to meaningfully participate in music. The study also sets the stage for research focused on the rehabilitation of holistic music experiences in hearing-impaired instrumentalists, rather than a sole focus on the restoration of specific auditory deficiencies. Future research in this area should place greater focus on the relationship between auditory deficits and the benefits of music listening and participation, and the degree to which worsening auditory deficits reduce those benefits.

Acknowledgments. The authors acknowledge contributions from Amy Wang for her support in the interview guide development and participant recruitment and from Ewan Macpherson and Susan Scollie for reviewing an earlier version of the manuscript. The authors also thank Marjorie Leek for sharing her survey questionnaire.

\section{REFERENCES}

Alexander JM. (2013) Individual variability in recognition of frequency-lowered speech. Semin Hear 34(2):215-218.

Allsup RE, Benedict C. (2008) The problems of band: an inquiry into the future of instrumental music education. Philos Music Educ Rev 16(2):156-173.

Amlani AM, Punch JL, Ching TYC. (2002) Methods and applications of the audibility index in hearing aid selection and fitting. Trends Amplif 6(3):81-129.

Arehart KH, Kates JM, Anderson MC. (2011) Effects of noise, nonlinear processing, and linear filtering on perceived music quality. Int $J$ Audiol 50(3):177-190.

Barry CA, Britten N, Barber N, Bradley C, Stevenson F. (1999) Using reflexivity to optimize teamwork in qualitative research. Qual Health Res 9(1):26-44.

Bartel LR, Greenberg S, Friesen LM, Ostroff J, Bodmer D, Shipp D, Chen JM. (2011) Qualitative case studies of five cochlear implant recipients' experience with music. Cochlear Implants Int 12(1):27-33.

Bentler R, Chiou L. (2006) Digital noise reduction: an overview. Trends Amplif 10(2):67-82.

Bentler R, Wu Y-H, Kettel J, Hurtig R. (2008) Digital noise reduction: outcomes from laboratory and field studies. Int J Audiol 47(8):447-460.

Berger R. (2015) Now I see it, now I don't: researcher's position and reflexivity in qualitative research. Qual Res 15(2):219-234.

Chasin M, Hockley NS. (2014) Some characteristics of amplified music through hearing aids. Hear Res 308:2-12.

Chasin M, Russo FA. (2004) Hearing aids and music. Trends Amplif 8(2):35-47.

Chisolm TH, Johnson CE, Danhauer JL, Portz LJP, Abrams HB, Lesner S, McCarthy PA, Newman CW. (2007) A systematic review of health-related quality of life and hearing aids: final report of the American Academy of Audiology task force on the health-related quality of life benefits of amplification in adults. $J$ Am Acad Audiol 18(2):151-183.

Coffman D, Adamek M. (1999) The contributions of wind band participation to quality of life of senior adults. Music Ther Perspect 28(1):27-40.

Coffman DD. (2002a) Music and quality of life in older adults. Psychomusicology 18(1-2):76-88.

Coffman DD. (2002b) Banding together: new Horizons in lifelong music making. J Aging Identity 7(2):133-143.

Coffman DD. (2009) Learning from our elders: survey of New Horizons International music association band and orchestra directors. Int J Community Music 2(2-3):227-240.

Coffman DD, Adamek MS. (2001) Perceived social support of New Horizons band participants. Contrib Music Educ 28(1):27-40.

Coffman DD, Levy KM. (1997) Senior adult bands music's new horizon: not only do senior adult bands benefit members, the 
community, and university students in a practical sense, they also bring joy to all involved. Music Educ J 84(3):17-22.

Conrad CF, Gunter R. (2000) To be more useful: embracing interdisciplinary scholarship and dialogue. New Dir Higher Educ 110: $49-62$.

Contrera KJ, Betz J, Li L, Blake CR, Sung YK, Choi JS, Lin FR. (2016) Quality of life after intervention with a cochlear implant or hearing aid. Laryngoscope 126(9):2110-2115.

Creswell JW. (2007) Qualitative Inquiry \& Research Design. 2nd ed. Thousand Oaks, CA: Sage Publications.

Croghan NBH, Arehart KH, Kates JM. (2014) Music preferences with hearing aids: effects of signal properties, compression settings, and listener characteristics. Ear Hear 35(5): e170-e184.

Dabback WA. (2008) Identity formation through participation in the Rochester New Horizons band programme. Int $J$ Community Music 1(2):267-286.

Emiroglu S, Kollmeier B. (2008) Timbre discrimination in normalhearing and hearing-impaired listeners under different noise conditions. Brain Res 1220:199-207.

Feder K, Michaud D, Ramage-Morin P, McNamee J, Beauregard Y. (2015) Prevalence of hearing loss among Canadians aged 20 to 79: audiometric results from the 2012/2013 Canadian health measures survey. Health Rep 26(7):18-25.

Feldmann H, Kumpf W. (1988) Musikhoren bei Schwerho rigkeit mit und ohne Horgerat [Listening to music in the hard-of-hearing individual with and without hearing aid]. Laryng Rhinol Otol 67(10):489-497.

Finefter-Rosenbluh I. (2017) Incorporating perspective taking in reflexivity: a method to enhance insider qualitative research processes. Int J Qual Methods 16(1):1-11.

Fulford R, Ginsborg J, Goldbart J. (2011) Learning not to listen: the experiences of musicians with hearing impairments. Music Educ Res 13(4):447-464.

Fulford R, Ginsborg J, Greasley A. (2012) Hearing aids and music: the experiences of D/deaf musicians. Proceedings of the Ninth Triennial Conference for the European Society for the Cognitive Sciences of Music. Manchester, UK, August 17-22, 2015.

Gabrielsson A, Sjögren H. (1979) Perceived sound quality of sound-reproducing systems. J Acoust Soc Am 65(4):1019-1033.

Gates JT. (1991) Music participation: theory, research, and policy. Bull Counc Res Music Educ 109:1-35.

Gentles SJ, Jack SM, Nicholas DB, Mckibbon KA. (2014) Critical approach to reflexivity in grounded theory. Qual Rep 19(25):1-14.

Golafshani N. (2003) Understanding reliability and validity in qualitative research. Qual Rep 8(4):597-607.

Gough B, Madill A. (2012) Subjectivity in psychological science: from problem to prospect. Psychol Methods 17(3):374-384.

Guba EG. (1981) Criteria for assessing the trustworthiness of naturalistic inquiries. Educ Commun Technol 29(4):75-91.

Higgins P, Searchfield G, Coad G. (2012) A comparison between the first-fit settings of two multichannel digital signal-processing strategies: music quality ratings and speech-in-noise scores. $A m J$ Audiol 21:13-21.
Hsieh HF, Shannon SE. (2005) Three approaches to qualitative content analysis. Qual Health Res 15(9):1277-1288.

Jenstad LM, Van Tasell DJ, Ewert C. (2003) Hearing aid troubleshooting based on patients' descriptions. J Am Acad Audiol 14(7): 347-360.

Jutras PJ. (2011) The benefits of New Horizons band participation as self-reported by selected New Horizons band members. Bull Council Res Music Educ 187:65-84.

Kirchberger MJ, Russo FA. (2015) Development of the adaptive music perception test. Ear Hear 36(2):217-228.

Kirchberger MJ, Russo FA. (2016) Dynamic range across music genres and the perception of dynamic compression in hearing-impaired listeners. Trends Hear 20:1-16.

Knudsen LV, Laplante-Lévesque A, Jones L, Preminger JE, Nielsen C, Lunner T, Hickson L, Kramer SE. (2012) Conducting qualitative research in audiology: a tutorial. Int J Audiol 51(2):83-92.

Leek MR, Molis MR, Kubli LR, Tufts JB. (2008) Enjoyment of music by elderly hearing-impaired listeners. J Am Acad Audiol 19(6):519-526

Looi V, McDermott H, McKay C, Hickson L. (2008) Music perception of cochlear implant users compared with that of hearing aid users. Ear Hear 29(3):421-434.

Madsen SMK, Moore BCJ. (2014) Music and hearing aids. Trends Amplif 0(0):1-29.

Miles MB, Huberman MA. (1994) Qualitative Data Analysis: An Expanded Sourcebook. 2nd ed. Thousand Oaks, CA: Sage Publications.

Moore BCJ. (2016) Effects of sound-induced hearing loss and hearing aids on the perception of music. J Audio Eng Soc 64(3):112-123.

Moore BCJ, Tan C-T. (2003) Perceived naturalness of spectrally distorted speech and music. J Acoust Soc Am 114(1):408-419.

O’Toole P. (1994) I sing in a choir but I have "no voice"!. Q J Music Teach Learn 4-5(5-1):65-77.

Patton M. (2002) Qualitative Evaluation and Research Methods. 3rd ed. Thousand Oaks, CA: Sage Publications.

Rohwer D, Coffman D. (2006) Relationships between wind band membership, activity level, spirituality, and quality of life in older adults. Res Perspectives Music Educ 10(1):21-27.

Said EA. (2017) Health-related quality of life in elderly hearing aid users vs. non-users. Egyptian J Ear, Nose, Throat Allied Sci 18(3): 271-279.

Scollie S, Levy C, Pourmand N, Abbasalipour P, Bagatto M, Richert F, Moodie S, Crukley J, Parsa V. (2016) Fitting noise management signal processing applying the American Academy of Audiology pediatric amplification guideline: verification protocols. $J$ Am Acad Audiol 27(3):237-251.

Shenton AK. (2004) Strategies for ensuring trustworthiness in qualitative research projects. Educ Inf 22(2):63-75.

Sikolia D, Biros D, Mason M, Weiser M. (2013) Trustworthiness of grounded theory methodology research in information systems. Proceedings of the Eighth Midwest Association for Information Systems Conference, Normal, IL, May 24-25, 2013.

Small C. (1988) Musicking: The Meaning of Performing and Listening. Hanover, NH: Wesleyan University Press of New England. 
Strachan J. (2016) With Resound Enzos I Can for the First Time Appreciate Music. http://hearmystory.resound.com/resound-enzosi-can-first-time-appreciate-music/ Accessed August 4, 2016.

Uys M, van Dijk C. (2011) Development of a music perception test for adult hearing-aid users. South Afr J Commun Disord 58:19-47.

Vaisberg JM, Folkeard P, Parsa V, Froehlich M, Littmann V, Macpherson EA, Scollie S. (2017) Comparison of Music Sound Quality between Hearing Aids and Music Programs. AudiologyOnline, Article 20872. www.audiologyonline.com. Accessed August 17, 2017.

van Buuren RA, Festen JM, Houtgast T. (1999) Compression and expansion of the temporal envelope: evaluation of speech intelligibility and sound quality. J Acoust Soc Am 105(5): 2903-2913.
Van Den Hoonard DK. (2012) Qualitative Research in Action: a Canadian Primer. Don Mills, ON: Oxford University Press.

Watt D. (2007) On becoming a qualitative researcher: the value of reflexivity. Qual Rep 12(1):82-101.

Wilkinson S. (1988) The role of reflexivity in feminist psychology. Wom Stud Int Forum 11:493-502.

Zakis JA. (2016) Music perception and hearing aids. In: Popelka GR, Moore BCJ, Fay RR, Popper AN, eds. Hearing Aids. Cham, Switzerland: Spring International Publishing Switzerland, pp. 217-252.

Zimmerman E, Lahav A. (2012) The multisensory brain and its ability to learn music. Ann New York Acad Sci 1252(1):179-184. 